\title{
MICROWAVE ELECTROMAGNETIC FILED AND TEMPERATURE DISTRIBUTION IN A MULTILAYERED WOOD-CEMENT BOARD
}

\author{
Jaroslav Franek * Mojmír Kollár* — Ivan Makovíny ${ }^{* *}$
}

\begin{abstract}
The aim of this work was theoretical considerations of a multi-layer structure and estimation of some properties of previously designed and prepared modified wood-cement boards. These were enriched by carbon powder as electric admixture to gain a high damping of electromagnetic radiation in microwave frequency region. The dissipated power and temperature assessment based on the heat and electromagnetic wave propagation in case of perpendicular incident radiation are presented. As a practical criteria of the shielding effectiveness - the drop of the temperature measured behind an irradiated multilayered shield was utilized.

Keywords: wood-cement boards, multi-layer structure, heat and electromagnetic wave propagation, damping efficiency
\end{abstract}

\section{INTRODUCTION}

By using effective shields, unwanted influence of occasional or systematically acting electromagnetic fields on biological bodies or technological objects, can be suppressed and even eliminated. Although the metals are currently used as the shielding material, specific role can here play also non-metallic composites as polymers and particularly those with considerable electrical conductivity. Among prospective materials one can include also wooden composite boards with electrically conducting admixtures.

The goal, preceding this work, was to design and prepare modified wood-cement boards with a high damping of electromagnetic radiation in microwave frequency region. To attain this as the electric admixture - carbon powder was chosen. Among the advantages of Carbon one can figure in the chemical stability, low specific mass, relatively low price and the fact the Carbon is nontoxic. Sample boards were fabricated with different concentration of the C powder: $0 ; 5 ; 15$ a 25 (wt \%) of the wood mass, various type of wood particles and their different density. The specific conductivity of $\mathrm{C}$ is $\gamma=10^{-2} \mathrm{Sm}^{-1}$ and typical (average) dimensions of particles used, were: area $-3,7 \mu \mathrm{m}^{2}$, length $2.1 \mu \mathrm{m}$, and width $1 \mu \mathrm{m}$.

During preparation of the mixture to be pressed into a wood-cement board, at the beginning the cement + water + Carbon were stirred together, and wooden wool or splinters were admixed later. Afterwards the boards 1000 $\times 300 \mathrm{~mm}$ of thickness from 8 to $10 \mathrm{~mm}$ were prepared by pressing the mass in a mould. The principal prescript for a board fabrication was even mass of wooden-wool (WW) $100 \mathrm{~g}$ and cement (CE) $100 \mathrm{~g}$ in (W) $200 \mathrm{~g}$ of water and the amount of $4 \mathrm{~g}$ of admixture - Calcium-chloride $\left(\mathrm{CaCl}_{2}\right)$.

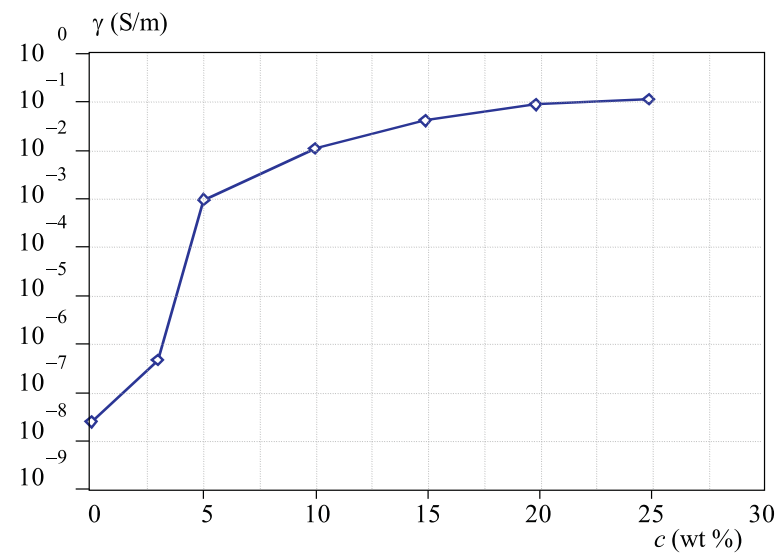

Fig. 1. Dependence of electric conductivity of C-modified woodcement composite on $\mathrm{C}$ concentration

\section{PHYSICAL CONSIDERATIONS}

Favourable damping properties of wooden materials - plywood, woodchip with modified electric proprieties by using conductive admixtures ( $\mathrm{Cu}$-powder and other metallic-colloid dilutions) are found in literature [1]-[4]. Wood cement composites (WCC) are fabricated, similarly like plywood and woodchip boards as large-area board construction materials. They are mostly used as the cladding material for the building facing due to their good acoustic and warm insulating properties. By using electrically conductive admixture - carbon-powder, added

\footnotetext{
* Faculty of Electrical Engineering and Information Technology, Department of Electromagnetic Theory, Ilkovičova 3 , 81219 Bratislava, Slovakia, jaroslav.franek@stuba.sk; ${ }^{* *}$ Faculty of Environmental and Manufacturing Technology, Department of Woodworking Machines and Equipment, Technical University in Zvolen, T. G. Masaryka 24, 96053 Zvolen, Slovakia, makoviny@vsld.tuzvo.sk
} 
Table 1. Relevant parameters of individual layers

\begin{tabular}{cccccc}
\hline $\begin{array}{c}\text { WCC } \\
\text { board }\end{array}$ & $\begin{array}{c}\mathrm{C} \\
\mathrm{wt} \%\end{array}$ & $\begin{array}{c}\varepsilon^{\prime} \\
(-)\end{array}$ & $\begin{array}{c}\varepsilon^{\prime \prime} \\
(-)\end{array}$ & $\begin{array}{c}\rho \\
\mathrm{kg} / \mathrm{m}^{3}\end{array}$ & $\begin{array}{c}\lambda \\
(\mu \mathrm{W} / \mathrm{mm} \mathrm{K})\end{array}$ \\
\hline $\mathrm{a}$ & 0 & 4.30 & 0.1 & 0.1 & - \\
$\mathrm{b}$ & 5 & 4.50 & 0.1741 & 350 & $<100$ \\
$\mathrm{c}$ & 10 & 6.66 & 0.1882 & - & 120 \\
$\mathrm{~d}$ & 15 & 4.85 & 0.1804 & 700 & 150 \\
$\mathrm{e}$ & 25 & 6.20 & 0.2920 & 1200 & 300 \\
\hline
\end{tabular}

In frequency range $2-3 \mathrm{GHz}$

to a Portland cement bond, one can due to increased electric conductivity improve the damping factor of the WCC board regarding the electromagnetic radiation.

Basic damping characteristic of a shielding material is the so called total insertion loss ie the ability to impede the electromagnetic wave propagation through the shielding layer. The insertion loss is usually measured for perpendicularly incident radiation.

The total insertion damping or the effectivity of a shield is attributed to two different effects - due to reflection, and due to absorption.

The designed shielding WCC structure has displayed relatively good reflection properties, because of conducting admixture (Carbon content varying from 5 to $25 \mathrm{wt}$ $\%$ ). On the other hand, the absorption characteristics are not so easy to be understood without a more detailed insight into the structure - what was also the aim of this work.

\section{EXPERIMENT}

To get useful information about the energy absorbed in individual layers - it was thought [5] of measuring not only the temperature difference at the respective sides of a shielding structure, but also inside of this. To provide such a measurement holes of different depths were

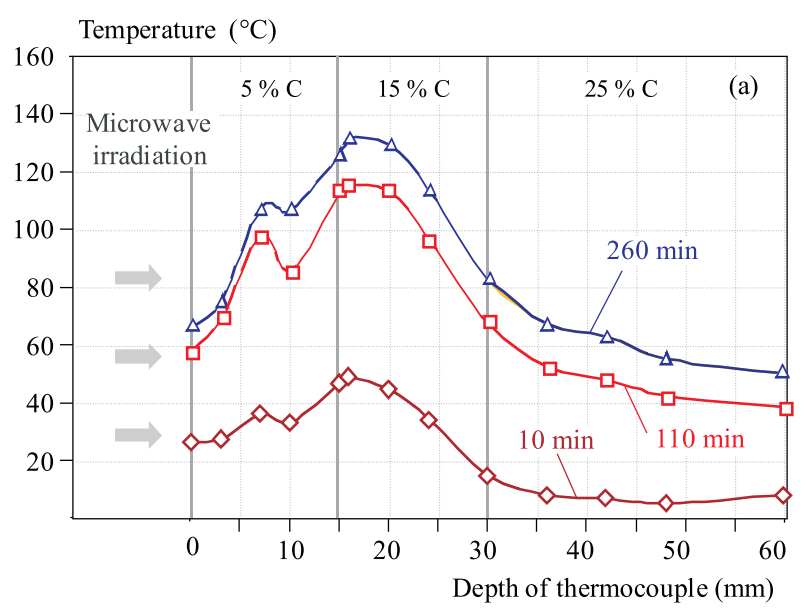

drilled in shielding WCC boards as indicated in Fig. 2., and the inside temperature was measured using a set of thermocouple-sensors. The temperature of irradiated and the other side of the multilayered structure was measured by a pyrometer.

Results of typical measurement are shown in Fig. 3 in two cases - with and without a metal plate "behind" the WCC structure. It was believed that placing of a metallic sheet would provide a better defined boundary conditions due to forced (practically total) reflection of transmitting waves. However, see Fig. 3, the differences were not significant. Except, that there was noticed a small peak or a "wavy" character closer to a surface on which the waves were incident (probably due to higher reflection at the end and consequent interference) in case with the bottom metal plate in Fig. 3(a).

More striking feature is that it took quite a long time until a heat steady-state was attained - as can be seen from the same figure, for three different times of microwave irradiation (10, 110 and 260 minutes).

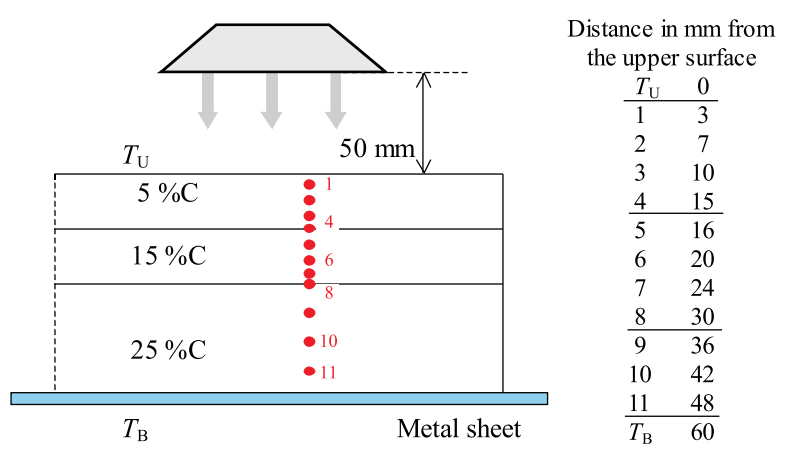

Fig. 2. Experimental set-up: Layers of WWC boards exposed to microwave radiation at Magnetron frequency of $2.45 \mathrm{GHz}$ (approximately $700 \mathrm{~W}) ; 1$ - to 11 measuring points (thermocouples), $T_{H}$ temperature of upper and $T_{S}$ bottom surface were measured by a pyrometer.

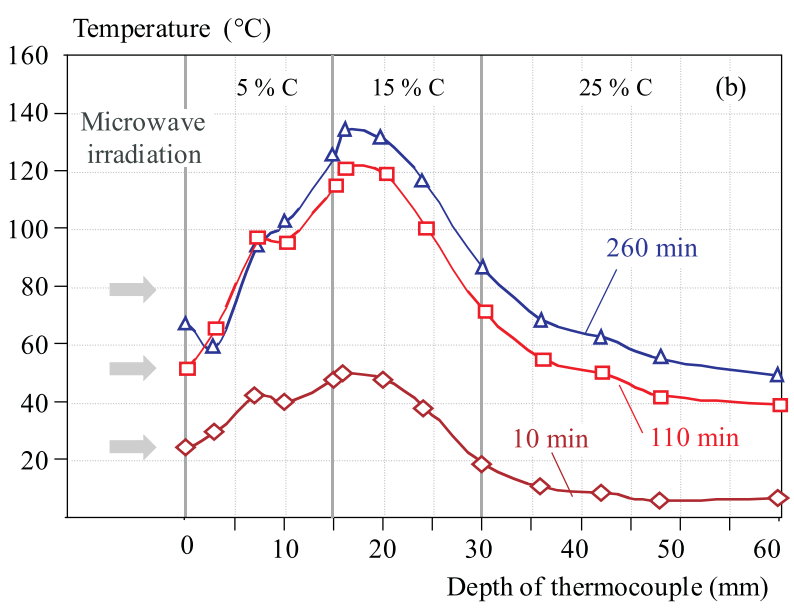

Fig. 3. Measured temperatures after 10, 110 and 260 minutes (steady state); (a) - with a metal sheet at the bottom, (b) - without a metal sheet at the bottom 


\section{ANALYTICAL MODEL}

The calculation of the wave transmission and reflection through and from a sandwiched multi-layers was conducted step by step, beginning from the bottom layer (in Fig. 4) named as Layer 1. This was ended by a metal sheet of a thickness exceeding the so called penetration depth (as well in experiments as in calculations) to have defined boundary conditions. Each of $n$ layers was described by its characteristic $Z_{0 n}$, load $Z_{2 n}$ and input $Z_{1 n}$ impedances and wave propagation constants $\gamma_{n}$, defined as

$$
\begin{gathered}
Z_{0 n}=\sqrt{\frac{j \omega \mu_{0}}{j \omega \varepsilon_{0}\left(\varepsilon_{n}^{\prime}-j \varepsilon_{n}^{\prime \prime}\right)}} \\
\gamma_{n}=\frac{j \omega}{c} \sqrt{\varepsilon_{n}^{\prime}-j \varepsilon_{n}^{\prime \prime}}
\end{gathered}
$$

where $\varepsilon_{n}^{\prime}$ and $\varepsilon_{n}^{\prime \prime}$ are the real and imaginary components of the complex relative premittivity $\tilde{\varepsilon}_{n}=\varepsilon_{n}^{\prime}-j \varepsilon_{n}^{\prime \prime}$ of the $n$-th layer, and $\varepsilon_{0}, \mu_{0}$ are the universal vacuum constats ( $c=\frac{1}{\sqrt{\varepsilon_{0} \mu_{0}}}-$ is velocity of the light in free space). Here $\omega=2 \pi f$ stands for angular frequency with $f$ being the frequency of electromagnetic waves. It was anticipated that permittivity can be considered as frequency independent in actual microwave range.

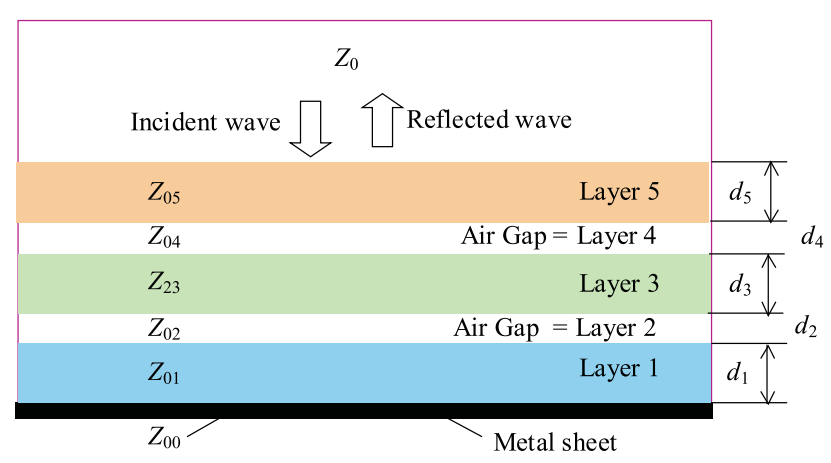

Fig. 4. Calculation model: Even layers $(1,3,5)$ are characterized by wave impedances $Z_{01}, Z_{03}, Z_{05}$ of individual WCC boards, while impedances $Z_{02}, Z_{04}$ - of odd layers (air gaps) are that of free-space (air), $Z_{0}=377 \Omega$.

The input impedance $Z_{11}$ of Layer 1 , being loaded by impedance $Z_{12}$ - which is the input impedance of thick enough conducting plate (with characteristic impedance even at microwave frequency $Z_{00} \rightarrow 0$ ), is

$$
Z_{11}=Z_{01} \tanh \left(\gamma_{1} d_{1}\right)
$$

Here $Z_{01}$ is the characteristic impedance of Layer 1, given by (1) for $n=1$.

It is now easy to express the input impedance of $n$-th layer (for $n>1$ ) using that of preceding (n-1) layer

$$
Z_{1 n}=Z_{0 n} \frac{Z_{0 n} \sinh \left(\gamma_{n} d_{n}\right)+Z_{1 n-1} \cosh \left(\gamma_{n} d_{n}\right)}{Z_{0 n-1} \cosh \left(\gamma_{n} d_{n}\right)+Z_{0 n} \sin \left(\gamma_{n} d_{n}\right)}
$$

note here, the frequency-dependent terms - as predicted by (1) and (2).

In even layers from $n=1,2, \ldots, 5$ (ie except $n=0$ ) - there are the WCC boards, while for odd $n$-s there are "intentionally introduced effective air gaps" - due to imperfect contact of the WCC boards (bending, surface roughness, etc ).

Now, in individual layers $(n)$ the electromagnetic waves will be described in frequency domain (using the complex representation of electric and magnetic fields) expressed by a sum of forward $E_{F n}, H_{F n}$ and backward $E_{B n}, H_{B n}$ waves

$$
\begin{aligned}
& E_{n}(\xi)=E_{F n}(0) e^{-\gamma_{n} \xi}+E_{B n}(0) e^{\gamma_{n} \xi} \\
& H_{n}(\xi)=H_{F n}(0) e^{-\gamma_{n} \xi}-H_{B n}(0) e^{\gamma_{n} \xi}
\end{aligned}
$$

where

$$
H_{F n}(0)=\frac{E_{F n}(0)}{Z_{0 n}} \quad H_{B n}(0)=\frac{E_{B n}(0)}{Z_{0 n}}
$$

It should be stressed that only the normal incidence and reflection is considered here due to a complexity of oblique propagation with respect to the surfaces, not only in calculations but also in measurement. Further, the power flow density per unit area (Poynting) in individual layers will be given as

$$
P_{n}(\xi)=\frac{1}{2} E_{n}(\xi) H_{n}^{*}(\xi)
$$

where $H^{*}$ denotes a complex conjugate of $H$. In (5) to $(8), \xi$ - refers to a local, to surface perpendicular coordinate and in each of layers: $\xi \in\left(0, d_{n}\right)$.

Finally, we can determine the volume power loss density $h_{n}(\xi)$ (the heat dissipation density) in each of the WCC layers from the real part of (8) as

$$
h_{n}(\xi)=-\frac{\mathrm{d}}{\mathrm{d} \xi} \operatorname{Re}\left\{P_{n}(\xi)\right\}
$$

The electromagnetic field now can determined in all layers $(n=1,2, \ldots, 5)$ providing the value of one of $E, H$ components is known at an arbitrary point. Notwithstanding this, the reduced dependences can be derived taking the reference level for instance at the surface of the primary incident wave (the top of Layer 5).

The heating of individual boards due to absorption of electromagnetic energy can be estimated on the basis of a simple one dimensional thermal equation, in certain aspect of a similar kind that is the electromagnetic wave equation solved by (5) or (6)

$$
\Delta T_{n}-\frac{1}{\lambda} \rho_{n} c_{n} \frac{\mathrm{d} T_{n}}{\mathrm{~d} t}=-\frac{h_{n}(\xi)}{\lambda}
$$

where $\lambda_{N}$ - is the thermal conductivity $(\mathrm{W} / \mathrm{m} \cdot \mathrm{K}), \rho_{N}$ - is the density, and $c_{N}$ - is the specific heat capacity of 


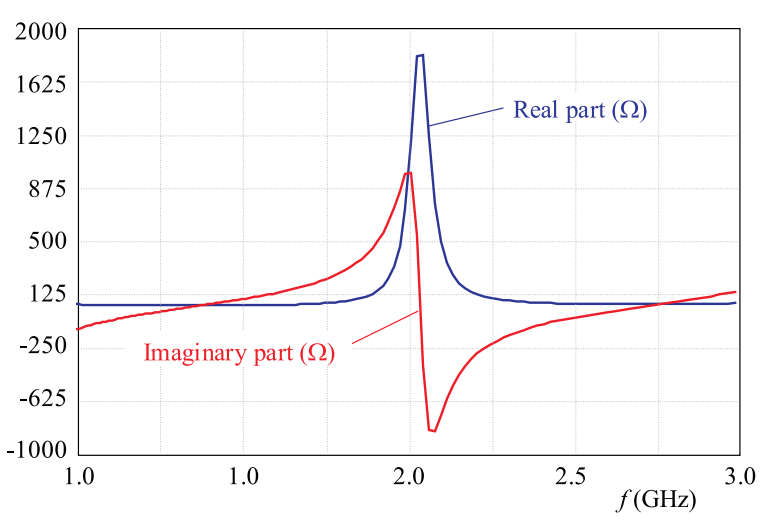

Fig. 5. Frequency dependence of the input impedance at Layer 3 of the structure from Fig. 4; Real and Imaginary parts

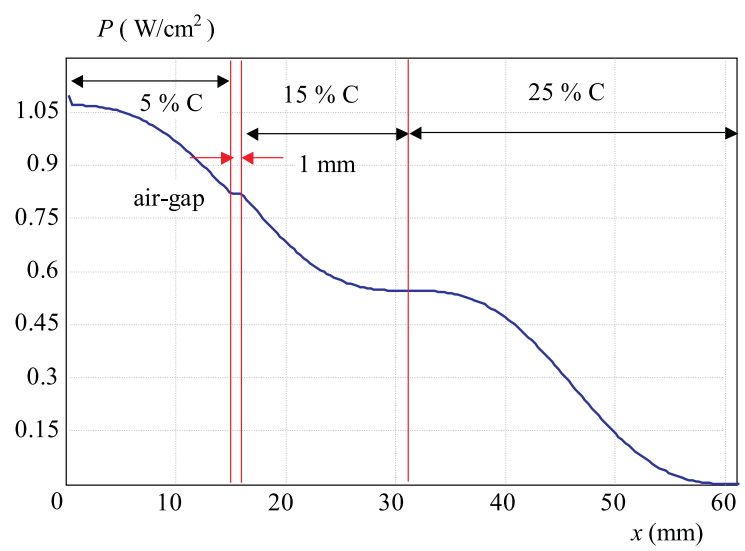

Fig. 7. Active part of the complex (Poynting) power flow density per unit area, as calculated from (8) across the WCC sandwich (containing $1 \mathrm{~mm}$ air-gap) at $2 \mathrm{GHz}$

individual layers. This, in one dimensional and stationary state $\mathrm{d} / \mathrm{d} t=0$, leads to

$$
\frac{\mathrm{d}^{2} T_{n}(\xi)}{\mathrm{d} \xi^{2}}=-\frac{h_{n}(\xi)}{\lambda_{n}}
$$

and two boundary conditions are to be met for an unambiguous solution of this equation in each of $N$ individual layers - that means one needs to determine $2 N$ constants. To express the solution in the sandwiched structure shown in Fig. 4, let us now introduce new space variable $x$ with the origin placed at the top of it so that

$$
\begin{array}{lll}
\text { Layer 5: } & x \in\left(x_{0}, x_{1}\right) & x_{1}=d_{5} \\
\text { Layer 4: } & x \in\left(x_{1}, x_{2}\right) & x_{2}=x_{1}+d_{4} \\
\text { Layer 3: } & x \in\left(x_{2}, x_{3}\right) & x_{3}=x_{2}+d_{3} \\
\text { Layer 2: } & x \in\left(x_{3}, x_{4}\right) & x_{4}=x_{3}+d_{2} \\
\text { Layer 1: } & x \in\left(x_{4}, x_{5}\right) & x_{5}=x_{4}+d_{1}
\end{array}
$$

above $x_{0}=0$, and clearly $x_{5}=\sum_{n=1}^{5} d_{n}$

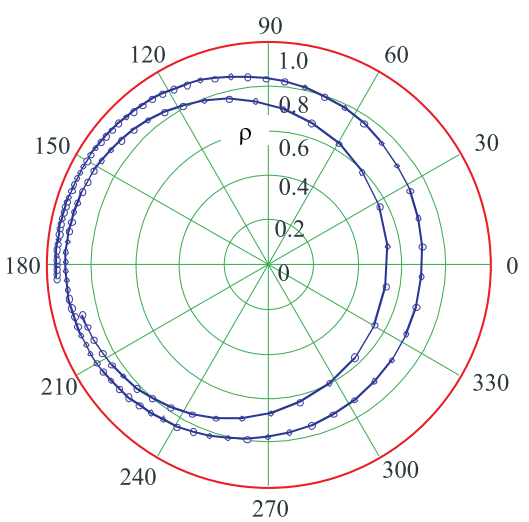

Fig. 6. Complex diagram of reflection coefficient in frequency rane 1-3 $\mathrm{GHz}$

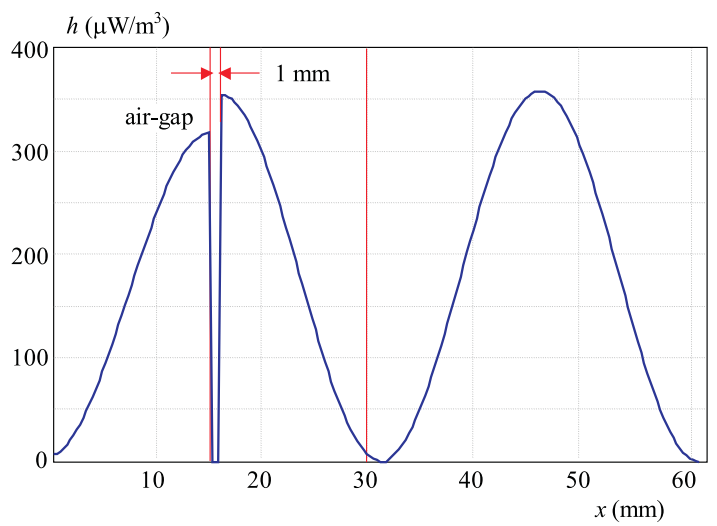

Fig. 8. The volume density of dissipating power as given by (9) is the "source" distribution function for the heat evaluation, note the effect of the included air-gap

The heat flow density $\left(\chi_{n}\right)$ at each of $N+1$ edges is proportional to temperature gradient,

$$
\begin{gathered}
\chi_{n}=-\left.\lambda_{n} \frac{\mathrm{d} T(x)}{\mathrm{d} x}\right|_{x_{N-n}} \\
n=N, N-1, \ldots, 0
\end{gathered}
$$

In a steady-state the difference of the heat flow densities at the bounding surfaces is equal to the integral of the power density "generated" in a given layer per unit area

$$
\begin{aligned}
\Delta \chi_{n} & =\chi_{n}-\chi_{n-1}=\int_{x_{N-n}}^{x_{N-n+1}} h_{n}(x) \mathrm{d} x \\
n & =N, N-1, \ldots, 1
\end{aligned}
$$

It can be verified that

$$
\sum_{n=1}^{N} \Delta \chi_{n}=\chi_{N}-\chi_{0}=\int_{x_{0}}^{x_{N}} h_{n}(x) \mathrm{d} x=\Delta \chi_{N}
$$

is equal to total energy dissipating per unit area in the sandwiched layers. From (11) and (13) we have

$$
\Delta \chi_{N}=-\left.\lambda_{N} T^{\prime}\right|_{0}+\left.\lambda_{0} T^{\prime}\right|_{N}
$$




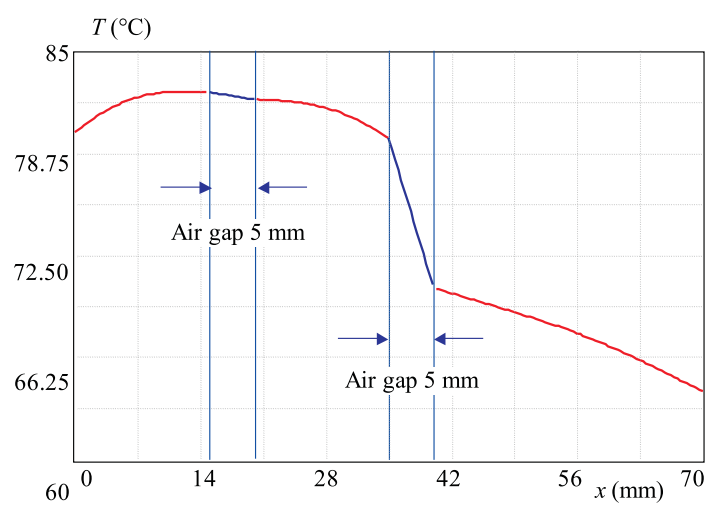

Fig. 9. Calculated temperature distribution in WCC sandwich containing two wide air-gaps, $5 \mathrm{~mm}$, frequency of irradiation 2.45 $\mathrm{GHz}$, power at the top of sandwich $15 \mathrm{~mW} / \mathrm{cm}^{2}$.

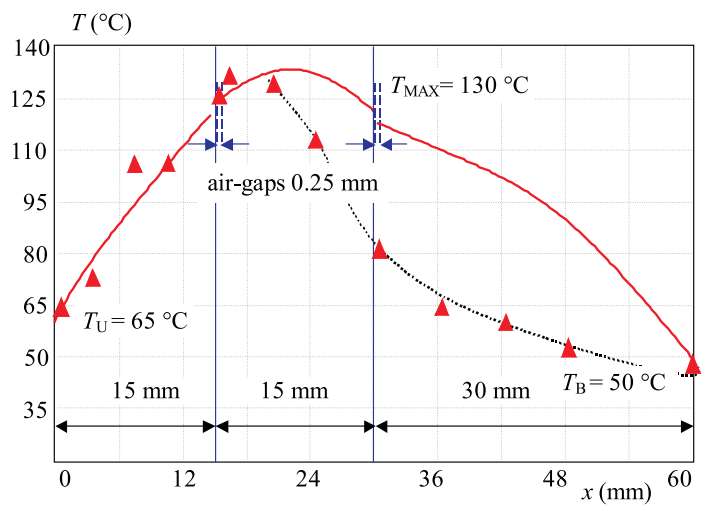

Fig. 11. Calculated temperature distribution in WCC sandwich containing two negligible air-gaps, $0.25 \mathrm{~mm}$, frequency of irradiation $2.45 \mathrm{GHz}$, power at the top of sandwich $150 \mathrm{~mW} / \mathrm{cm}^{2}$. Triangles are measured points from Fig. 3(a) after 260 minutes of irradiation.

when an abbreviated notation was used

$$
\left.\frac{\mathrm{d} T(x)}{\mathrm{d} x}\right|_{x_{k}}=\left.T^{\prime}\right|_{k}
$$

Let us now put

$$
\left.T^{\prime}\right|_{0}=\epsilon \frac{\Delta \chi_{N}}{\lambda_{N}},\left.\quad T^{\prime}\right|_{N}=(\epsilon-1) \frac{\Delta \chi_{N}}{\lambda_{0}}
$$

providing (14) will remain valid for any $\epsilon \leq 1$. Then it is easy to find

$$
\begin{aligned}
\left.T^{\prime}\right|_{k} & =\frac{1}{\lambda_{N-k}}\left[\epsilon \Delta \chi_{N}-\sum_{\nu=N+1-k}^{N} \Delta \chi_{\nu}\right] \\
k & =0,2, \ldots, N .
\end{aligned}
$$

The last expression gives for $k=N$ the temperature gradient at the edge of the sandwiched structure (the bottom in Fig. 4) and is related to the heat flow leaving the structure for outer environment with thermal conductivity $\lambda_{0}$. In the investigated structure $N=5$, and the bottom metal plate can be considered as having $\lambda_{0} \rightarrow \infty$ and thus $\left.T^{\prime}\right|_{5} \rightarrow 0$.

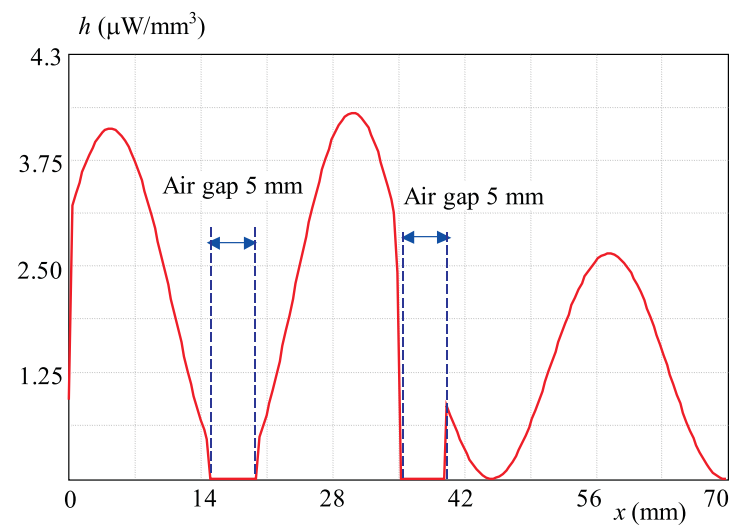

Fig. 10. Calculated heat dissipation density (9) in case from Fig. 9; with two $5 \mathrm{~mm}$ air-gaps, frequency of irradiation $2.45 \mathrm{GHz}$, power at the top of sandwich $15 \mathrm{~mW} / \mathrm{cm}^{2}$.

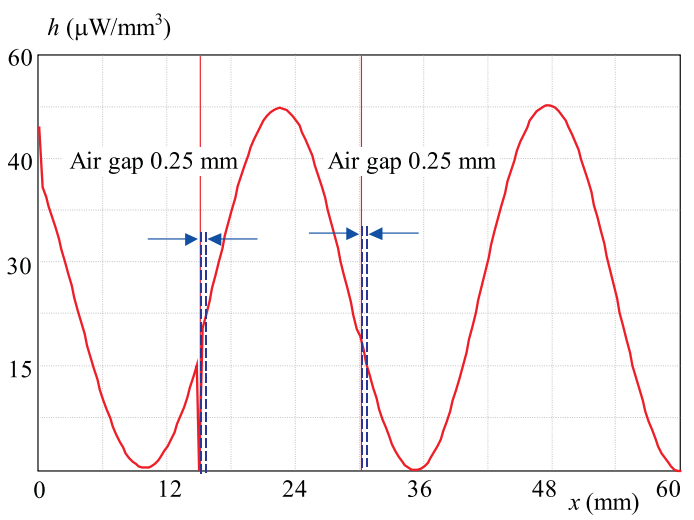

Fig. 12. Calculated heat dissipation density (9) in case from Fig. 11; with two $0.25 \mathrm{~mm}$ air-gaps, $0.25 \mathrm{~mm}$, frequency of irradiation $2.45 \mathrm{GHz}$, power at the top of sandwich $150 \mathrm{~mW} / \mathrm{cm}^{2}$.

Irrespectively of the ending plate, at least we need to determine 5 derivatives $\left.T^{\prime}\right|_{0}$ to $\left.T^{\prime}\right|_{4}$ that can be done iteratively using (16), but the value of $\epsilon$ must bev known. Further, there are 4 planes where the continuity of the temperature $i e:\left.T\right|_{1},\left.T\right|_{2},\left.T\right|_{3},\left.T\right|_{4}$, is obvious. And finally, if the temperatures $T_{U}$ and $T_{B}$ will determined experimentally - altogether we have 11 conditions for matching the unknowns - 10 constants required for solution of differential equation (10) and parameter $\epsilon$ in equations (16).

$\mathrm{Al}$ calculations according (1) to (9) and concerning the electromagnetic field in sandwiched structure from Fig. 4 were done in one MathCad programme, the rest related to the heat transfer in other programme, built to solve the task in MatCad environment, as well. Results of the electromagnetic field penetration are illustrated in Fig. 5 to Fig. 8.

\subsection{Electromagnetic field calculation}

The calculation of electromagnetic field is conducted in "reversed" order, from bottom to top as the individual layers are numbered. This is because to evaluate the 
input impedance of any layer one has to know its loading conditions. In Fig. 5., there is shown for instance the frequency dependence of the input impedance at Layer 3 (real and imaginary part). It is obvious that in given frequency range the input impedance is very sensitive to the frequency of irradiation. Note that the dependence shown in Fig. 5. belongs to a a part of the sandwiched structure (from Fig. 4.) including Layer 3 (WCC board "d"), Layer 2 (air), Layer 1 (WCC board "e") and the metal plate at the bottom. For material parameters of above layers see the Tab. 1, [6].

Properties of investigated WCC structure, in frequency range from $1 \mathrm{GHz}$ to $3 \mathrm{GHz}$, are documented in Fig. 6, where the reflection coefficient $\rho$ calculated for a wave incident at the top is given. In case of total reflection the points would lay on a circle with radius 1 , while in the case of total absorption all of them would by placed in exactly in the middle of the graph $(\rho=0)$.

After evaluating the field vectors (5), (6) in individual layers, the complex power flow pre unit area (Poynting) is obtained from (8) and the course of its real part (the active power flow per unit area) though the whole shielding structure is shown in Fig. 7. The volume density of dissipating power as given by (9) is in Fig. 8 and this is the "source" distribution function for the heat transfer evaluation.

\subsection{The heat transfer calculation}

Results of the heat distribution as calculated in WCC multi-layer structure are in Fig. 9 to Fig. 12. In fact the three layer structure (boards containing 5, 15 and $25 \mathrm{wt} \%$ oc C) were treated as a five layered structure taking into consideration that while stacking the boards one can not exclude more or less affecting air-gaps between them due to surface roughness, bending of the boards etc. The effect of such a gaps on the temperature distribution is shown in Fig. 9 and Fig. 10 together with the appropriate heat dissipation density in Fig. 11 and Fig. 12.

It is obvious that air-gaps can substantially affect the resulting absorption. The character of experimental dependence at $2.45 \mathrm{GHz}$ from Fig. 3 is close to that calculated and shown in Fig. 11 at least in that both are showing maxima of the temperature distribution closely behind the first board (at approximately $20 \mathrm{~mm}$ from the top surface). At present it is not completely clear why the tails of experimental and calculated temperature curves are so different concerning their slope ant the temperature gradient at the bottom surface. This should by clarified by further investigation, however it can be verified that in case of a finite dimension disk (contrary to the calculated infinitely large slab case) the concave character of the temperature curve will change to a convex one while the thickness to radius ratio of the considered disk decreases. By other words, the radial heat removal (not actual in a case of infinite slab) substantially influences the temperature distribution in a case of finite-dimension slabs.

\section{CONCLUSION}

New type of a WCC composite board structure was designed and fabricated with relatively high shielding efficiency. To better understand the absorption process in its multilayered structure theoretical analysis and a numerical solution of the microwave and thermal field was conducted. Calculated and experimentally observed characteristics were at least qualitatively in good agreement. It should be stressed that there is a lack of the WCC board parameters in frequency rage between $1 \mathrm{GHz}$ to $10 \mathrm{GHz}$ due to insufficiency of the appropriate measurement technique, and they had to be estimated to approximate values (used also in presented calculations). From different character of the tails of calculated and measured curves (uneven type of curvature) one can conclude that the process in the thickest board $(30 \mathrm{~mm})$ may be of a more complex nature.

\section{Acknowledgement}

This research was supported by Slovak Agency for uphold of the research and development under project APVV- 0229-06: Applied research of wood-cement boards for screening of the electromagnetic fields and for increasing of the fire resistance.

\section{REFERENCES}

[1] LI WANG, J. et al : Study on Influence factors of Wood-metal Composites Electromagnetic Shielding, Northeast Forestry University China, No. AP42 (2005), 185-188.

[2] LI YIXING, et al : Study on Medium Density Fiberboard made from Wood fieber/Cooper powder, 2005.

[3] NAGASAWA, et al : Electromagnetic Shielding Particleboard with Nickel-Plated Wood Particlel,, Journal of the Japan Wood research Society 35 No. 12 (1989), 1092-1099.

[4] NAGASAVA, CH.-UMEHARA, H.-KOSHIZAKI, N.-URABE, K.-KUMAGAI, Y.-KNABE, T: Effects od Wood Species on Electroconductivity and Electromagnetic Shielding Properties of Electrolessly Plated Sliced Veneer with Nickel, Journal of the Japan Wood Research Society 40 No. 10 (1994), 1092-1099.

[5] MAKOVÍNY, I. : Technical University in Zvolen, Scientific studies $2 / 1999 / \mathrm{B}$.

[6] SCOTT, A. W.: Understanding Microwaves. USA: New York, J. Willey and Sous, 1993.

[7] http://www.ptc.com/products/mathcad/.

Recieved 18 November 2009 